\title{
Studies on the morphology of leukaemic blast cells in relation to haematological parameters
}

\author{
D.M.Agase* \\ Govt. J.S.T.P.G.College, Balaghat, India \\ S.B.Zade \\ P.G.T.D. of Zoology, RTM Nagpur University, Nagpur, India \\ M.S.Markam \\ Govt. J.S.T.P.G.College, Balaghat, India \\ P.M. Mohurle \\ P.G.T.D. of Zoology, RTM Nagpur University, Nagpur, India \\ P.R. Chaudhari \\ P.G.T.D. of Zoology, RTM Nagpur University, Nagpur, India \\ S.P. Padole \\ D.D. Bhoyar College of Arts and Science Mouda, Nagpur, India \\ P.R. Bandebuche \\ D.D. Bhoyar College of Arts and Science Mouda, Nagpur, India \\ P.D. Bhoyar \\ D.D. Bhoyar College of Arts and Science Mouda, Nagpur, India \\ A.D.Pangal \\ D.D. Bhoyar College of Arts and Science Mouda, Nagpur, India \\ A.K. Ganju \\ Ganju Hematology Clinic and Hospital, Nagpur, India \\ *Corresponding author. E-mail: sbt.durgesh@gmail.com
}

\begin{abstract}
A combination of haematological parameters with morphological evaluation of peripheral blood and bone marrow blast cells is crucial for leukaemia diagnosis. FAB (FrenchAmerican-British) classification is a simple and powerful diagnostic tool for leukaemia in developing countries like India. Differentiation block in the early stages of haematopoiesis and morphological characteristics of leukemic blast cells are directly related to haematological parameters. The present study is an approach to increase understanding of the simple morphological FAB classification of leukaemia in relation to haematological parameters. The present study revealed that Chronic Myeloid Leukaemia (CML) was the most common type of leukaemia , followed by Acute Myeloid Leukaemia, Acute Lymphoid Leukaemia (ALL), and Chronic Lymphoid Leukaemia (CLL) in Nagpur. Most of the cases of Acute Leukaemia had severe anaemia and thrombocytopenia. Highest variation was found in Total WBCs count of different types of leukaemia, particularly in different subtypes of AML. The present study also suggested that FAB classification is not outdated, but it does require continuous revalidation and other procedures for refinement.
\end{abstract}

Keywords: Blast cell, Bone marrow, FAB classification, Haematopoiesis, Leukaemia

\section{INTRODUCTION}

Leukaemia is a malignant neoplasm of haematopoietic cells. Specific genetic events related to cellular differentiation and proliferation of hematopoietic cell contributes to malignant transformation of cells and their progeny forming clones of leukaemia cells. In leukaemia, differentiation block occurs in the early stages of hematopoiesis, thus resulting in undifferentiated cells are named as blast cells. In a few cases, cells other than blast cells are counted that are known as blast equivalent (Singh, 2018). Leukemic blast cell and other

\section{Article Info}

https://doi.org/10.31018/

jans.vi.2267

Received: April 29, 2020

Revised: May 18, 2020

Accepted: May 28, 2020

\section{How to Cite}

Agase, D.M. et al. (2020). Studies on the morphology of leukaemic blast cells in relation to haematological parameters. Journal of Applied and Natural Science, 12(2): $171-179$. https://doi.org/10.31018/

jans.vi.2267 blast equivalent demonstrate extra-ordinary biological, morphological, and clinical heterogeneity (Baviskar, 2016). The perspective of the classification and characterization of leukemic blast cell is imperative for the treatment of different subtypes of leukaemia because in the leukemic form, by definition the bone marrow must contain at least $20 \%$ blast cell and study of blast cell morphology is the first step in the diagnostic pathway (Sabina et al., 2014). The present study was carried out with the aim to know the morphological characteristics of blast cells of peripheral blood and bone marrow in relation to different haemato- 
logical parameters in newly diagnosed cases of leukaemia.

\section{MATERIALS AND METHODS}

The study was conducted at the Animal Physiology Laboratory, Department of Zoology RTM Nagpur University, Nagpur, and all samples were collected from the Ganju Hematology Clinic \& Hospital Nagpur and Kane Hematology and Oncology Laboratory Nagpur. In this study total numbers of 89 subjects were selected in which 10 were normal healthy individuals and 79 were leukemic patients who were divided into four subgroups: 31 were diagnosed as CML (Chronic Lymphoid Leukaemia ), 25 as AML (Acute Myeloid Leukaemia), 21 as ALL (Acute Lymphoid Leukaemia) and 2 were as Chronic Lymphoid Leukaemia (CLL). The case selection was based on clinical features. Informed consent was taken from all the patients and the study was ethically approved. Bone marrow aspirate and peripheral blood were collected from Ganju Hematology Clinic \& Hospital Nagpur and Kane Hematology and Oncology Laboratory Nagpur.

For haematological variables, samples were collected in EDTA coated purple-top test tube. Haematological parameters R.B.Cs, W.B.Cs, haemoglobin, and platelets were estimated by an Automated Haematological Analyzer (Swelab Alfa analyzer SN18766), and blast cells were counted by Hemocytometer.

The study comprised of peripheral blood smears and bone marrow aspirate blood smears. Minor modification in standard Leishman's stain was done by either incubating slide or buffer solution or both (Sareen et al., 2018). Leishman's stain was modified using phenol crystals and liquefied phenol (Fasakin et al., 2014).

\section{RESULTS AND DISCUSSION}

In the present study, out of 79 leukemic peripheral blood samples of the patients, $31(39.240 \%)$ were diagnosed as CML, $25(30.656 \%)$, as AML 21 (26.582\%), as ALL and $2(2.532 \%)$ were as CLL (Table 1). In our study, among all cases, CML had the highest, and CLL had the lowest incidence

Table 1. Type of leukaemia in patients.

\begin{tabular}{ccccc}
\hline Leukaemia Type & AML & ALL & CML & CLL \\
\hline No. of Patients & 25 & 21 & 31 & 2 \\
\hline Table 2. Haemoglobin level in leukaemia patients. \\
\hline Haemoglobin & \multicolumn{5}{c}{ No. of Patients } \\
\cline { 2 - 5 } level & AML & ALL & CML & CLL \\
\hline$<\mathbf{7 g m} \%$ & 20 & 15 & 04 & 00 \\
$\mathbf{7 - 1 0 ~ g m \%}$ & 04 & 03 & 18 & 02 \\
$>\mathbf{1 0}$ gm $\%$ & 01 & 03 & 09 & 00 \\
Total & 25 & 21 & 31 & 02 \\
\hline
\end{tabular}

Haemoglobin range values in normal persons: Male; $14 \mathrm{gm}$ $\%$-18 gm\%, Female; $12-15 \mathrm{gm} \%$ rate. The incidence of $C M L$ was noted the highest $(45.3 \%)$ and that was the lowest of CLL $(5.7 \%)$ in Delhi (Vasavada et al., 1962, Rani et al.,1982). Similar observations were noted in Chandigarh and other metro cities of India like Mumbai and Calcutta (Chatterjea et al., 1962, Radha et al., 2014, Ahirwar et al., 2018). In the present study, AML had the second-highest incidence rate followed by ALL among all classes of leukaemia . The incidence rate of ALL was higher than AML in South India (Prakash et al., 1981, Varghese et al., 1984). In our study, the cases of ALL were the third predominant. In the present study most frequent $\mathrm{FAB}$ subtype of $\mathrm{AML}$ was $\mathrm{M} 2$ which comprised of $9(36 \%)$ patients, whereas $M 0, M 1, M 3$, M4, M5, M6, M7 comprised of 1 (4\%), 2 (8\%), 5 $(20 \%), 1(4 \%), 2(8 \%), 3(12 \%), 2(8 \%)$ respectively. Tata Memorial Institute reported AML-M2 as the most common FAB subtype of AML (Ghosh et al., 2003). Similarly, In the L1 subtype of ALL comprised of $15(71.4 \%)$ patients, whereas L2 and L3 comprised $4(19.04 \%)$ and $2(9.52 \%)$ patients respectively. It has been seen that the spectrum of leukaemia varies in different geographical regions according to varying lifestyles, economic conditions, and poverty rates (Gunz., 1977). But, in Nagpur, the incidence of different types of leukaemia does not differ markedly with other metro cities of India. CML was a more common type of leukaemia followed by AML, ALL, and CLL. Anaemia is a constant feature in all classes of leukaemia . In the majority of cases, it is due to bone marrow infiltration leading to decreased production, decreased red cell life span, and autoimmune destruction. (Farzana et al., 2016).

In the present study, $80.00 \%$ of cases of AML and $71.42 \%$ of cases of ALL had less than $7 \mathrm{gm} \% \mathrm{Hb}$. In CML $12.90 \%$ of cases had less than $7 \mathrm{gm} \% \mathrm{Hb}$. In CLL both patients had mild anaemia with 9.02 and $8.92 \mathrm{gm} \% \mathrm{Hb}$ Count (Table 2). The mean haemoglobin count of all classes of leukaemia

Table 3. Total WBCs count in Leukaemia Patients.

\begin{tabular}{ccccc}
\hline WBCs counts & \multicolumn{4}{c}{ No. of Patients } \\
\cline { 2 - 5 } & AML & ALL & CML & CLL \\
\hline $4 \times 10^{3} / \mu \mathrm{l}-10 \times 10^{3} / \mu \mathrm{l}$ & 06 & 06 & - & - \\
$10 \times 10^{3} / \mu \mathrm{l}-100 \times 10^{3} / \mu \mathrm{l}$ & 15 & 14 & 04 & - \\
$100 \times 10^{3} / \mu \mathrm{l}-160 \times 10^{3} / \mu \mathrm{l}$ & 03 & 01 & 24 & 02 \\
$>160 \times 10^{3} / \mu \mathrm{l}$ & 01 & - & 03 & - \\
\hline
\end{tabular}

Total WBCs range values in normal persons: $4.8 \times 10^{3} / \mu \mathrm{l}-$ $10.8 \times 10^{3} / \mu \mathrm{l}$

Table 4. Total Platelet counts in leukaemia patients.

\begin{tabular}{ccccc}
\hline Platelet Counts & \multicolumn{4}{c}{ No. of Patients. } \\
\cline { 2 - 5 } & AML & ALL & CML & CLL \\
\hline $40 \times 10^{3} / \mu \mathrm{l}-100 \times 10^{3} / \mu \mathrm{l}$ & 21 & 20 & - & - \\
$100 \times 10^{3} / \mu \mathrm{l}-150 \times 10^{3} / \mu \mathrm{l}$ & 02 & 00 & 01 & - \\
$150 \times 10^{3} / \mu \mathrm{l}-350 \times 10^{3} / \mu \mathrm{l}$ & - & 01 & 01 & 02 \\
$350 \times 10^{3} / \mu \mathrm{l}-550 \times 10^{3} / \mu \mathrm{l}$ & 02 & - & 29 & - \\
\hline
\end{tabular}

Total Platelet range values in normal persons: $175 \times 10^{3} / \mu \mathrm{l}-$ $450 \times 10^{3} / \mu \mathrm{l}$ 
Agase, D.M. et al. / J. Appl. \& Nat. Sci. 12(2): 171 - 179 (2020)
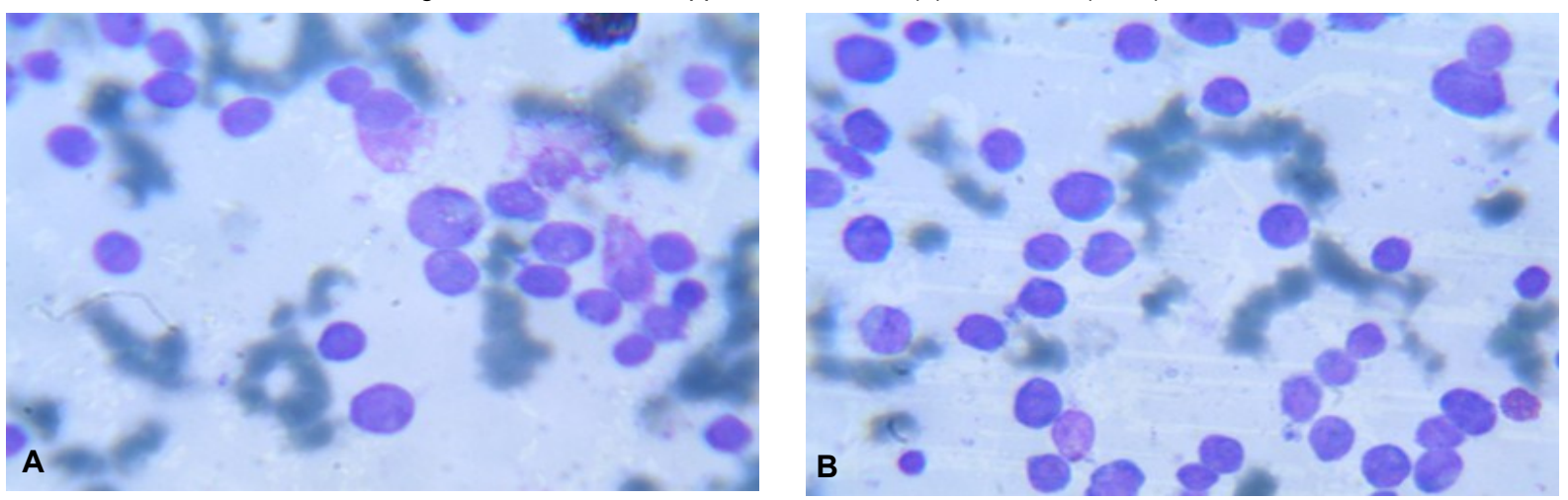

Fig. 1. AML-MO sub-type; $A$ and $B$. Bone marrow aspirate smear showing large and uniform blast cells, scanty cytoplasm, nucleus with open chromatin.
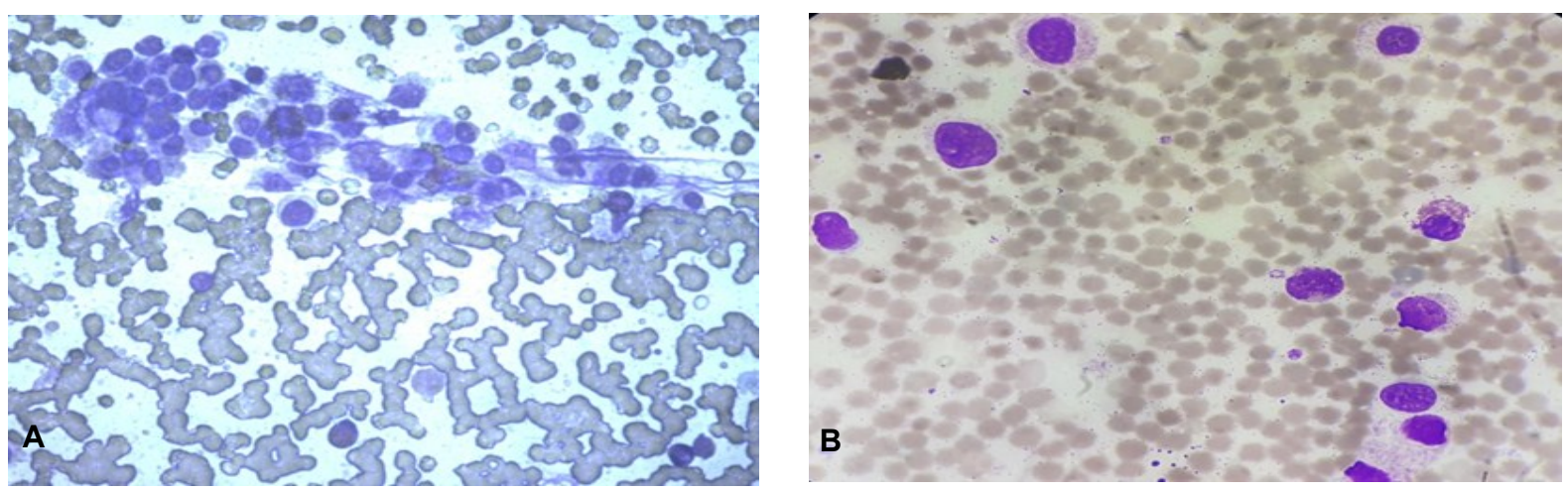

Fig. 2. AML-M1 sub-type; A-Bone marrow aspirate smear, B-peripheral blood smear; showing large blast cells with variable nucleocytoplasmic ratio, rare blast cell demonstrating Auer rods.
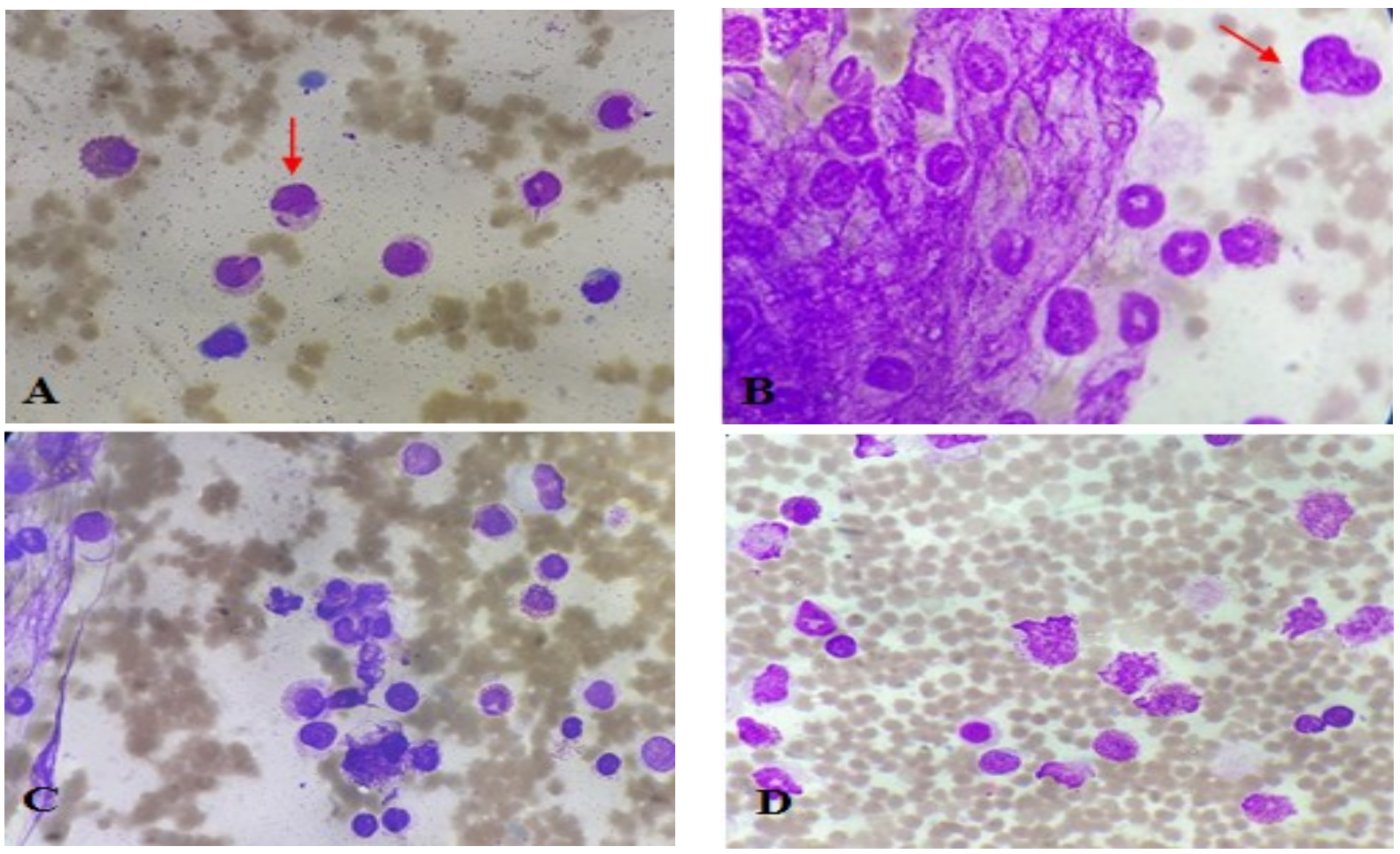

Fig. 3. $A M L-M 2$ sub-type; (Bone marrow aspirate smear (A,B,C and $D)$; showing large blast with oval and slightly indented nuclei and moderate amount of granulophilic cytoplasm. A-one of the blast showing an Auer rod (red arrow), B-one of the blast cell shows distinctive heart shape nucleus. $C$ and D-promyelocyte, myelocytes and metamyelocytes and neutrophils. $C$ and $D$ showing highest number of eosinophilic myelocytes. 

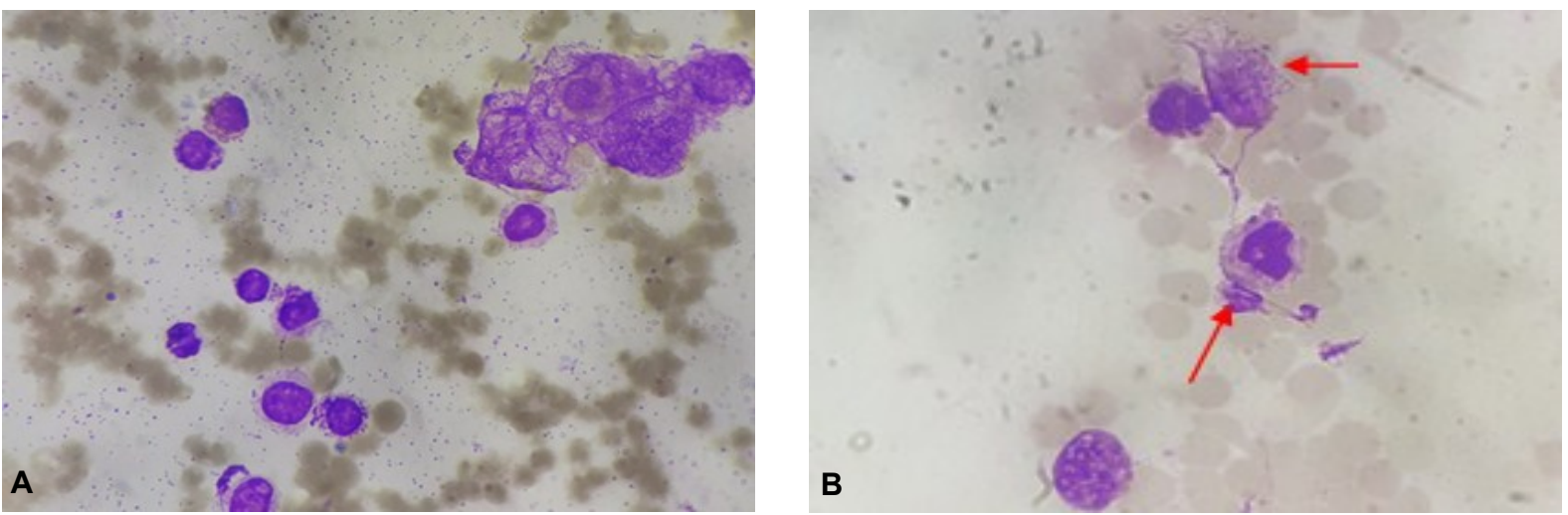

Fig. 4. AML-M3 sub-type; Bone marrow aspirate smear ( $A$, and $B)$ showing medium size Promyelocytes with densely packed coarse purple granular cytoplasm, Some cells showing auer rods in the form of bundle (Faggot cells)(red arrows), presence of Phi bodies in some cells (green arrow).
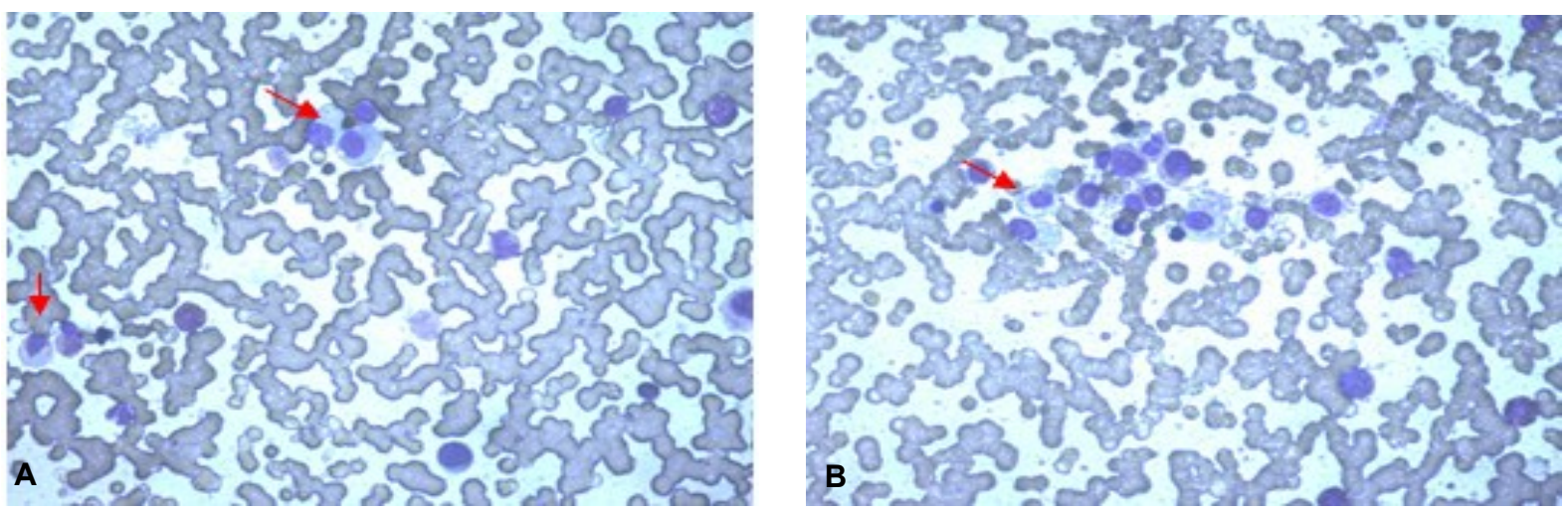

Fig. 5. AML-M4 sub-type. Peripheral blood smear (A, and $B$ ), showing medium size Myeloblast and Monoblast with lower nuclear to cytoplasmic ratio and agranular cytoplasm.
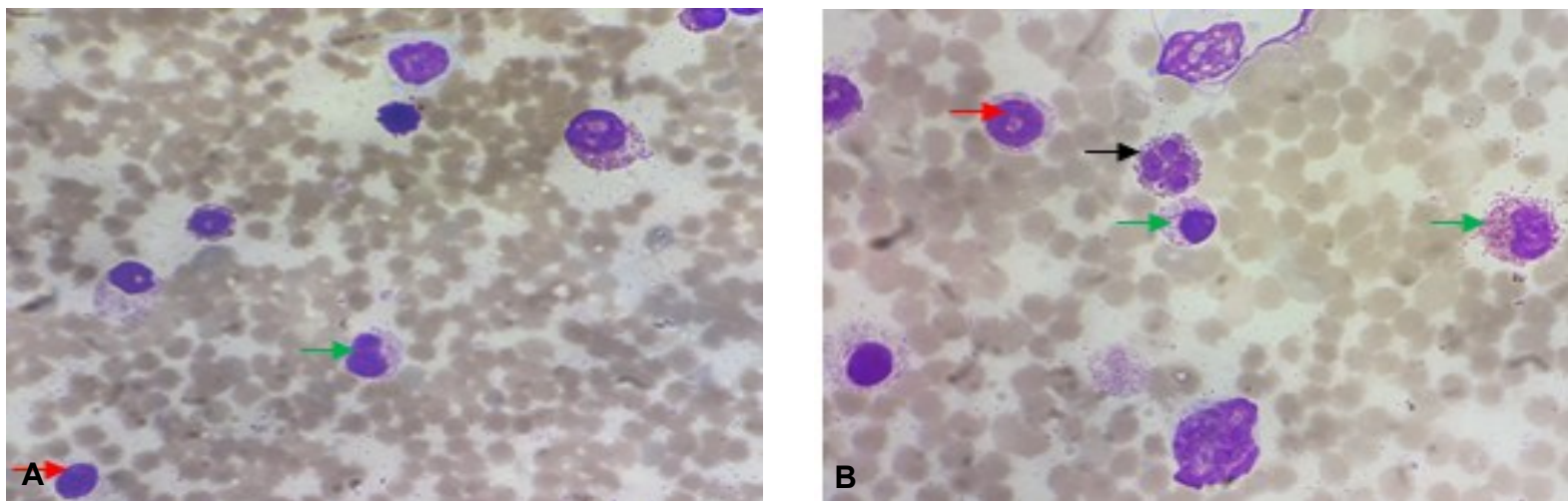

Fig. 6. AML-M5 sub-type. Peripheral blood smear (A,and B), showing blast cell (red arrow) and Promonocytes (green arrow) with lower N/C ratio, vacuolated cytoplasm. One cell showing apoptosis (black arrow).

was quite low, among them the most of the cases of acute leukaemia had severe anaemia. Other studies done by various authors also reported similar haemoglobin profiles of acute and chronic leukaemia patients in Hamidia Hospital, Bhopal (Ahirwar et al., 2015).

Leukocytosis is the common finding associated with all classes of leukaemia. In the present study, the total WBCs count of $77.41 \%$ cases of $\mathrm{CML}$ showed hyperleukocytosis (Table 3 ). Hyperleukocytosis is defined as a total peripheral WBC above $100 \times 10^{9} / \mathrm{L}$. Hyperleukocytosis increases the blood viscosity and is associated with the aggregation of leukemic cells in the microcirculation (Farzana et al., 2016). Thrombocytopenia is a well-known expression of acute leukaemias. In the present study, most of the cases of acute leukaemia had severed thrombocytopenia, whereas chronic leukaemia most of the cases had normal to moderate thrombocytopenia (Table 4). The association of bleeding with platelet count has been well documented in the literature by Gaydos et al. (1962) and was the first to document this finding in patients with acute leukaemia. They demonstrated 

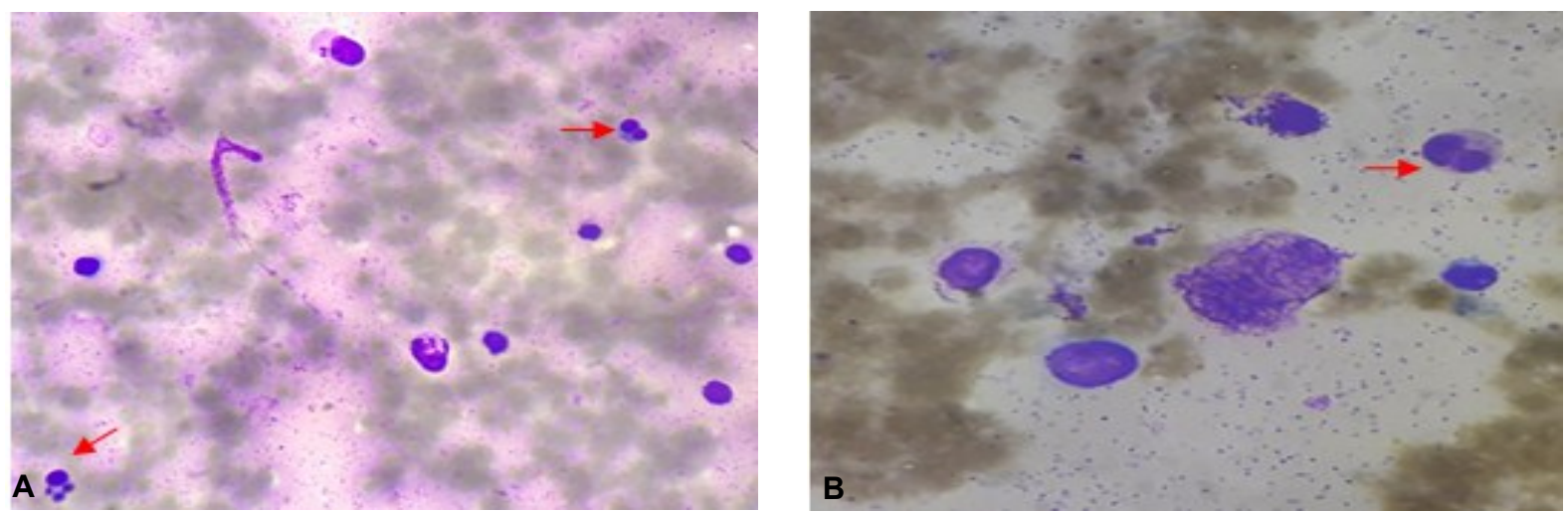

Fig. 7. AML-M6 sub-type; Peripheral blood smear (A, and B), showing blast cells with basophilic agranular cytoplasm. Some cells with two or more than two nuclei (red arrow).

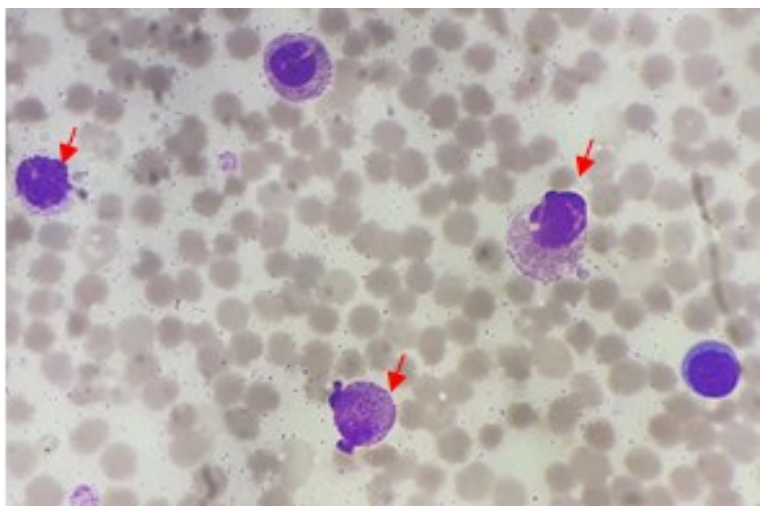

Fig. 8. AML-M7 sub-type; Peripheral blood smear $A$, showing Megakaryoblast with membrane budding (red arrow).

that there was a linear relationship between bleeding and low platelet count. The severity of thrombocytopenia in patients with leukaemia varies according to the type and stage of the disease. Patients with CML tend to have an elevated platelet count during the chronic phase and thrombocytopenia develops as the disease progresses from chronic to blastic phase. Thrombocytopenia is very common in the presentation in patients with acute leukaemia (AML and ALL). Many authors

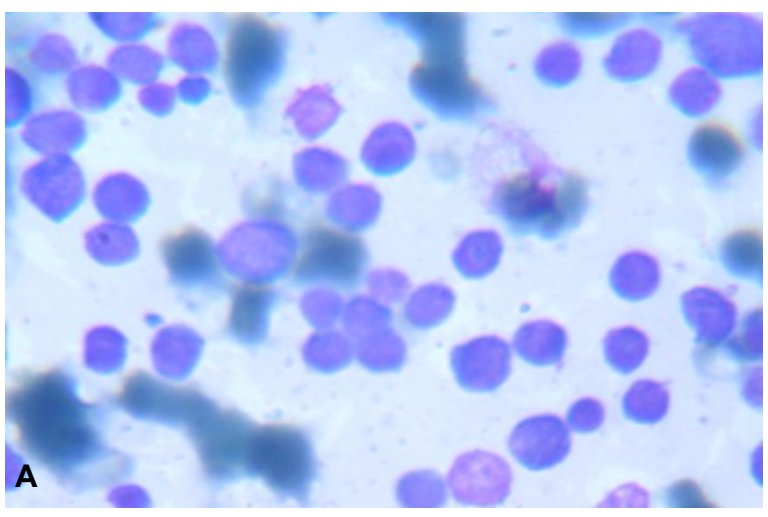

correlated the morphological feature of acute megakaryoblastic leukaemia (AML-M7) with thrombocytopenia. (Gaydos et al., 1962, Quazi., et al., 2002). These morphological features like platelet budding help in correctly diagnosing acute megakaryoblastic leukaemia (Sharma et al., 2009).

Morphological evaluation of peripheral blood smear and bone marrow cells is crucial for diagnosis and for the follow up in leukaemia. Therefore, microscopy with the combination of haematological parameters still remains a very important tool for diagnosis in leukaemia. In acute leukaemia, the classification now most widely used is the $F A B$ classification proposed in 1976, which is based on morphology. In addition to FAB Classification, several new classification systems have been proposed for the diagnosis of leukaemia.

FAB Classification stills an important and simple tool for leukaemia diagnosis in developing countries like India. But this classification requires continuous evaluation and refinement. The present study is an approach to understand how morphological characters concerning haematological parameters are important for the diagnostic classification of leukaemia. The FAB cooperative working group classified ALL into L1, L2, and L3 on the

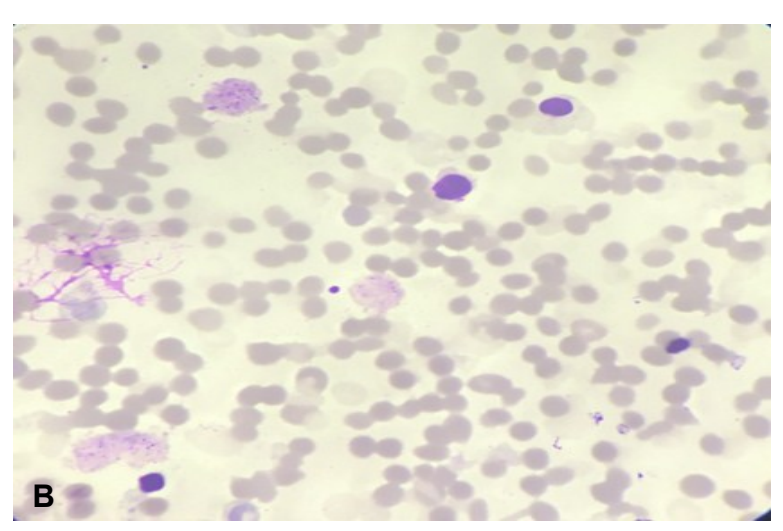

Fig. 9. ALL-L1 sub-type. A- Bone marrow aspirate smear, B-peripheral blood smear showing blast cells are small, uniform size, scanty cytoplasm, Nuclei have coarsely clumped chromatin, Inconspicuous 1-2 small Nucleoli. 

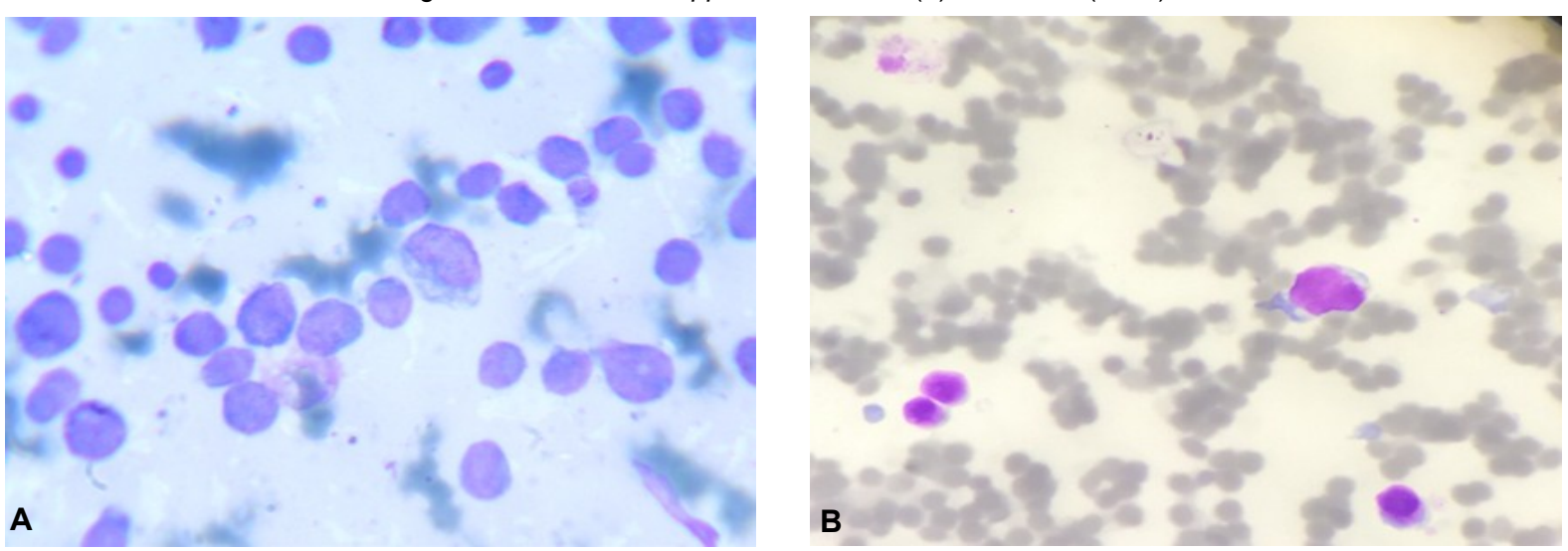

Fig.10. ALL-L2 sub-type; A-Bone marrow aspirate smear, B-peripheral blood smear showing blast cells are medium to large size, nuclei are variable in shape and size, 1-2 nucleoli, clumped chromatin, irregular nuclear membrane, moderate amount of basophilic cytoplasm.
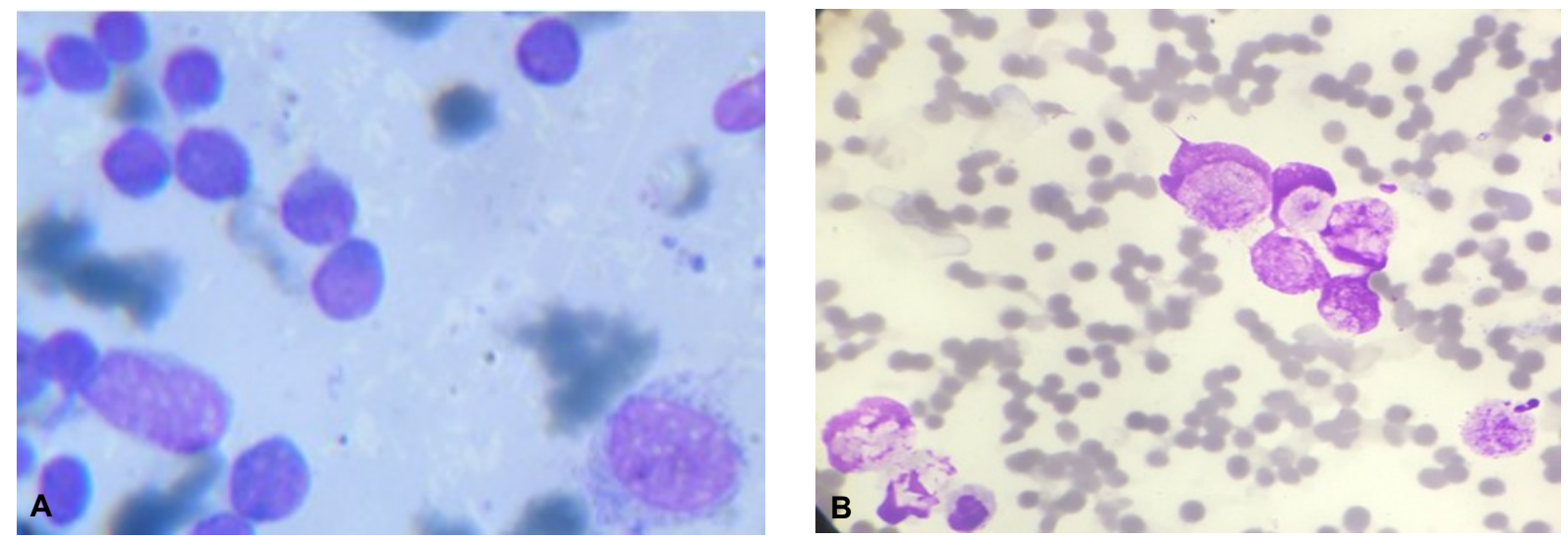

Fig. 11. ALL-L3 sub-type. A- Bone marrow aspirate smear, B-peripheral blood smear showing blast cells are intermediate to large size with clumped chromatin, prominent vacuoles, deeply basophilic cytoplasm with multiple vacuoles which also overlie the nucleus, prominent nucleolus.

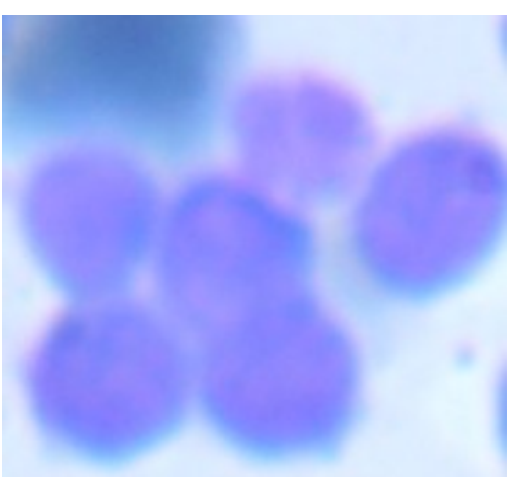

L1

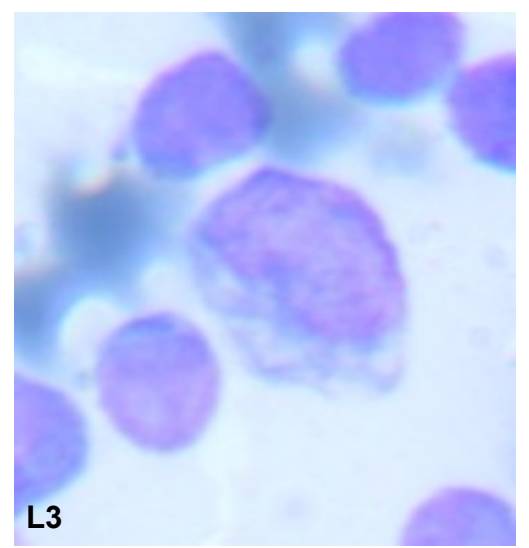

L3

Fig. 12. Showing comparative morphology of blast cell in sub-types of ALL.

basis of four morphological characteristics: N: C ratio, presences of nucleoli, characteristics of the nuclear membrane, and cell size (Bennettl et al., 1976).

The morphological characteristics of leukemic blast cells are given in Figs. 1-9. In our study, it was indicated that L1 lymphoblasts were small, uniform, and characterized by a high $\mathrm{N}$ : C ratio (Fig. 9). L2 lymphoblasts were medium to large size, heterogeneous, and lower $\mathrm{N}$ : C ratio (Fig. 10), while L3 lymphoblasts were heterogeneous and showed prominent vacuolation with deeply basophilic cytoplasm (Fig. 11). The study confirmed the work of authors who have also reported similar morphological characters in ALL patients (Conter et al., 2004, Sabina et al., 2014, Ladines et al., 2016).

Bone marrow aspirate smear of CLL patient 

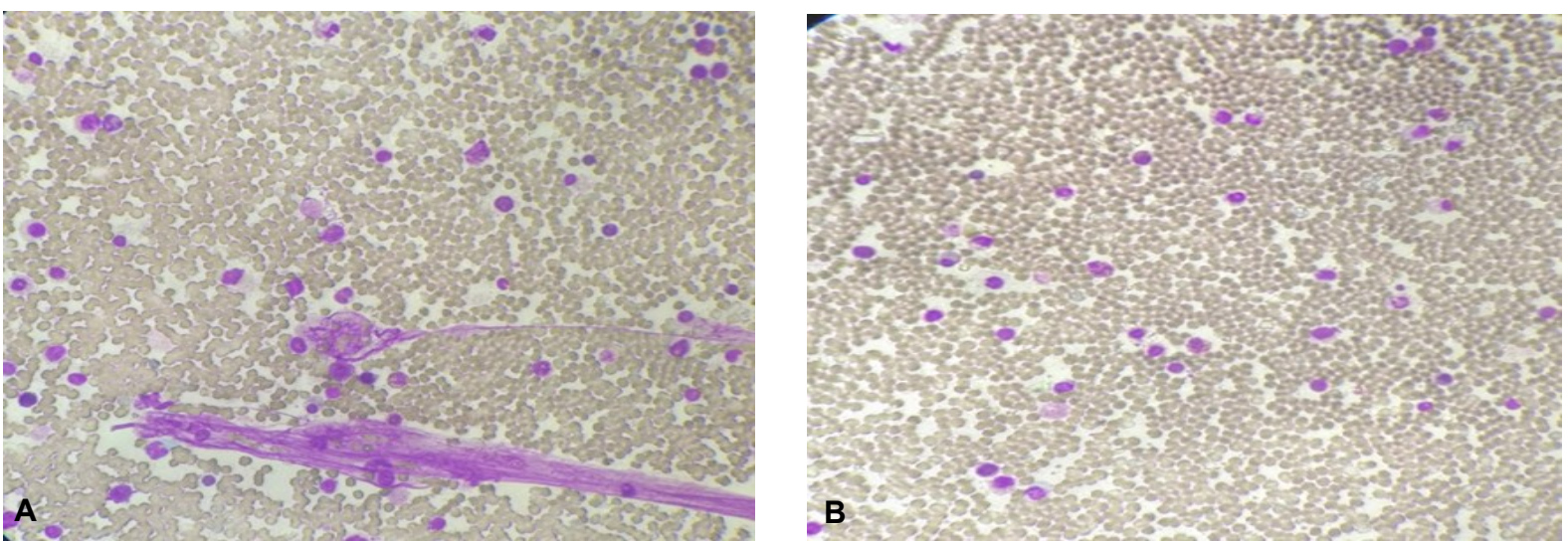

Fig. 13. $C M L, A$ and $B$, Bone marrow aspirate smear showing low power view showing leukocytosis, with all stages of myeloid cells from blast cell to neutrophils.
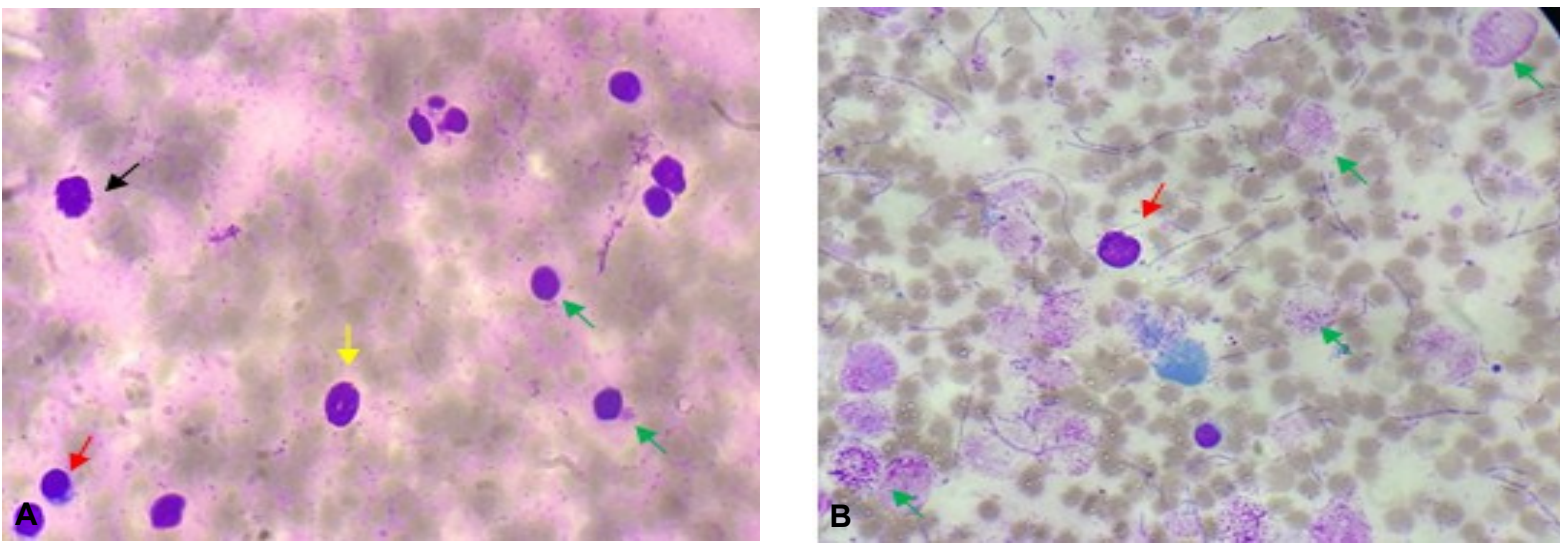

Fig. 14. CML A: Peripheral blood smear showing low power view showing leukocytosis with all stages of myeloid cells- myeloblast (green arrow), promyelocytes (red arrow) and myelocytes (yellow arrow), basophile made out by their coarse granules (black arrow), hyper granular basophiles. B: Peripheral blood smear showing large number of smudge cells (green arrow) and myelocyte bulge (red arrow).
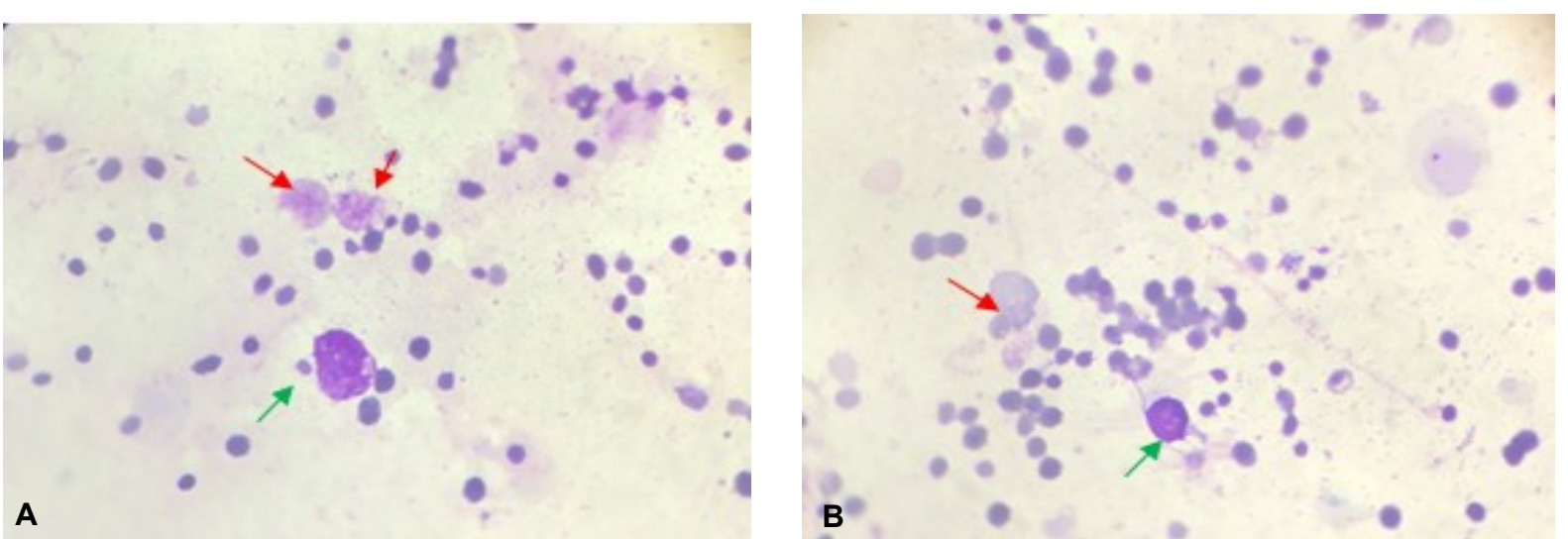

Fig. 15. CLL $A$ and B, Bone marrow aspirate smear low power view showing lymphocytosis, smudge cells (red arrow) and megakaryocyte (green arrow).

showed smudge cells and megakaryocytes (Fig. 15-17). Smudge cells were remnant cells that lacked any identifiable cytoplasmic membrane or nuclear structure. These smudge cells are also called basket cells (David et al., 2016). The peripheral blood smear of CML patient showed hyperleukocytosis with all stages of myeloid cells (Fig. 13-14). The present study confirmed the work of authors who have also reported similar hyperleukocytosis in CML patients (Karl et al., 2000, Danielle et al., 2008,) AML-M0 blast is difficult to diagnose the morphological point of view. In our study, AML-M0 myeloblasts were medium to large size with scanty cytoplasm and open chromatin nucleus (Fig. 1). AML-M1 myeloblasts were larger compared to the M0 blast and showed 


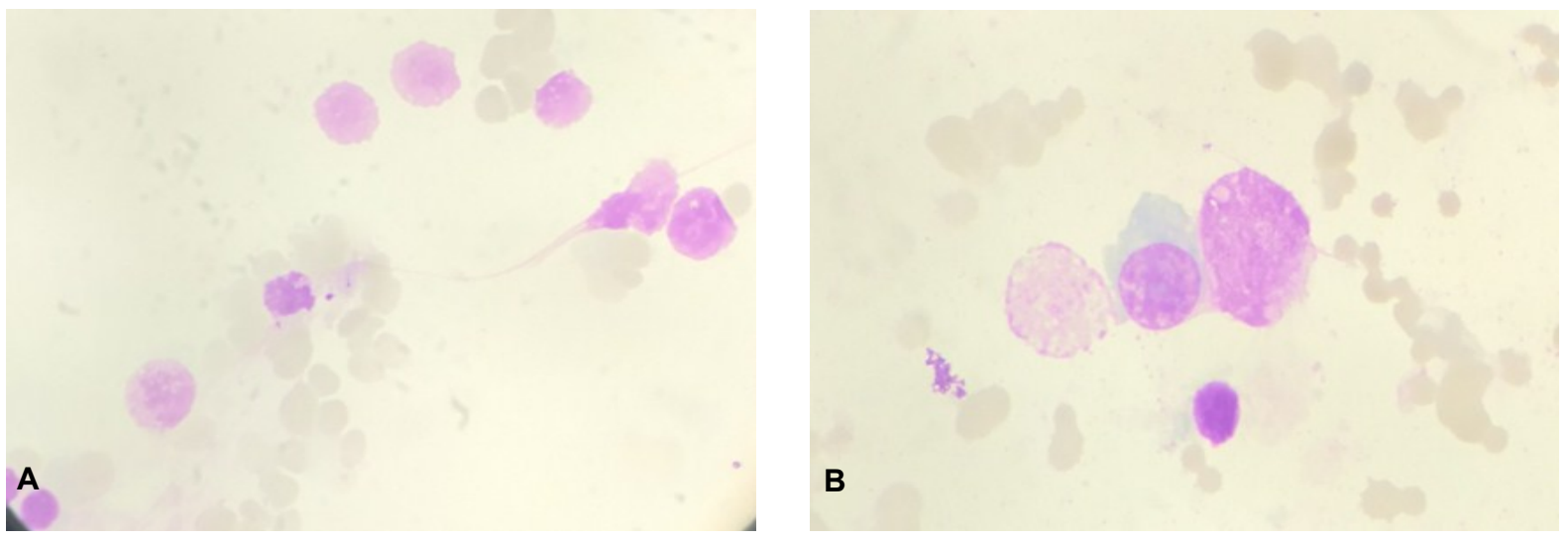

Fig. 16. CLL. ( $A$ and B, Bone marrow aspirate smear) showing smudge cells. Smudge cells are remnants of cells that lack any identifiable cytoplasmic membrane or nuclear structure. Smudge cells also called basket cells.
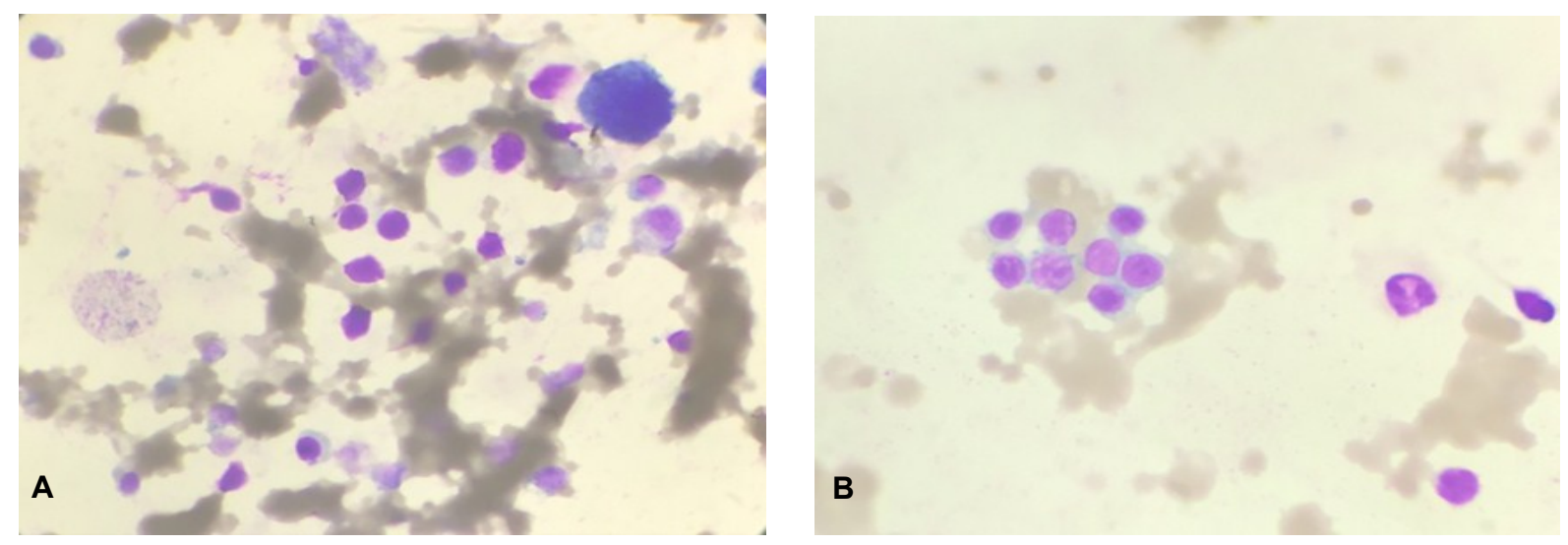

Fig. 17. CLL. A and B, Bone marrow aspirate smear showing leukemic Lymphocytes are uniform with round nucleus and cytoplasmic outline. Nuclear chromatin is coarsely clumped with no nucleoli. Large no. of smudge cells and some megakaryocyte. Abbreviations: AML: Acute myeloid leukaemia ; ALL: Acute lymphoblastic leukaemia ; CML: Chronic myeloid leukaemia ; CLL: Chronic lymphocytic leukaemia

variable nucleocytoplasmic ratio (Fig. 2). AML-M2 myeloblasts were large in size, oval in shape and contained a moderate amount of granulophilic cytoplasm. In our study, we found a distinct heart shape nuclear morphology in some AML-M2 blast (Fig. 3). In AML-M3 some promyelocytes show auer rods in the form of a bundle and Phi body (Fig. 4). AML-M4 myeloblast and monoblast were medium in size, low $\mathrm{N}$ : $\mathrm{C}$ ratio and contain agranular cytoplasm (Fig. 5). AML-M5 myeloblasts were characterized by peculiar vacuolated cytoplasm and some cells with apoptotic bodies (Fig. 6). Some AML-M6 blast contains more than two nuclei (Fig. 7). AML-M7 megakaryoblast showed membrane budding (Fig. 8). The most of our morphological characteristics of different subclasses of AML, except for AML-M2 and AML-M7, confirmed the finding of Ladines et al. (2016) in distinct heart shape nuclear morphology of AML-M2 blast and membrane budding of AML-M7 megakaryoblast have not been reported yet and supposed to be a new morphological feature.

\section{Conclusion}

The diagnosis is the first and most important step in the treatment of any disease. The present study is an approach to increase understanding of the simple morphological diagnostic $F A B$ classification of leukaemia in relation to haematological parameters. The FAB classification is replaced by $\mathrm{WHO}$. Still, FAB classification is a simple and powerful diagnostic tool in developing countries like India. The present study revealed that CML was the most common type of leukaemia, followed by AML, ALL, and CLL. In Nagpur, the incidence of different types of leukaemia does not differ markedly with other metro cities of India. Most of the cases of Acute Leukaemia had severe anaemia and thrombocytopenia. The highest variation found in Total WBCs count of different types of leukaemia, particularly in different subtypes of AML. The study concluded that FAB classification is a good guide for clinicians and hematologists. It is not outdated but it does require continuous revalidation and other procedures for refine- 
ment. Based on FAB morphology, a computeraided method is required for the diagnosis of specific subclasses of leukaemia. It is also required to correlate this abnormal morphological feature with prognostic approaches.

\section{REFERENCES}

1. Ahirwar R, Nigam R. K, Parmar D. (2018). A study of leukaemia's profile in central India. Tropical Journal of Pathology \& Microbiology, 4(2), Print ISSN: 24569887, Online ISSN: 2456-1487.

2. Baviskar J. (2016). Incidence of acute and chronic leukaemia $s$ in rural area at tertiary care teaching hospital: A five years of study. Indian Journal of Pathology and Oncology, 3(4), 710-713.

3. Bennett JM, Catovsky D, Daniel M-T, Flandrin G, Galton D. A. G, Gralnick H. R, Sultan C. (1976). Proposals for the classification of the acute leukaemias. British Journal of Haematology, 33(4), 451-458.

4. Chatterjea JB, Ghose S, Ray RN. (1962). Incidence of leukaemia. (An analysis of 544 cases studied in Calcutta). The Journal of the Association of Physicians of India, 10, 673-6.

5. Conter V, Rizzari C, Sala A, Chiesa R, Citterio M, Biondi A. (2004). Acute Lymphoblastic Leukaemia . Orphanet Encyclopedia.

6. Danielle E. W, Ling Z, Sophie S, Stephen L. (2008). Concurrent Megakaryocytic and Erythroid Chronic Myelogenous Leukaemia Blast Crisis. Arch Pathol Lab Med, 132, 1021-1025.

7. David O, Monica E, Estella M, Ricardo M, Jonathan C. Strefford, Daniel C. (2016). The morphology of CLL revisited: the clinical significance of prolymphocytes and correlations with prognostic/molecular markers in the LRF CLL4 trial. British Journal of Haematology, 174, 767-775.

8. Fasakin KA, Okogun GR, Omisakin CT, Adeyemi AA, Esan AJ. (2014). Modified Leishman Stain: The Mystery Unfolds. British Journal of Medicine \& Medical Research, 4(27), 4591- 4606.

9. Farzana C, Tahir S, Shamsi, Ali M. W. (2016). Clinical and Haematological Profile of Acute Myeloid Leukaemia (AML) Patients of Sindh. Journal of Hematology \& Thromboembolic Diseases, 4(2), 239.

10.Gaydos LA, Freireich EJ, Mantel N. (1962), The quantitative relation between platelet count and hemorrhage in patients with acute leukaemia . $N$ Engl $J$ Med, 266, 905-909.

11.Ghosh SC, Shinde SC, Kumaran GS, Sapre RS,
Dhond SR, Badrinath Y, Ansari R, Kumar A, Mahadik S, Chaugule AB, Nair CN. (2003). Hematologic and Immunophenotypic Profile of Acute Myeloid Leukaemia : An Experience of Tata Memorial Hospital. Indian J Cancer, 40(2), 71-76.

12. Gunz FW. (1977). The epidemiology and genetics of the chronic leukaemias. Clinics In Haematol, 6(1), 320.

13.Karl G, Daniel A. (2000). Chronic myelogenous leukaemia . Laboratory medicine, 31, 10.

14.Ladines-Castro W, Barragán-Ibã̃nez G, Luna-Pérez M.A, Santoyo-Sánchez A, Collazo-Jaloma J, Mendoza-García E, Ramos-Pe nafiel C.O. (2016), Morphology of Leukaemia s. Rev Med Hosp Gen Mex, 79(2), 107-113.

15.Prakash S, Ramamurthi, Gopalan R, Aurora AL. (1981). Leukaemias at Pondicherry. Indian J Cancer, 18 (1), 1-6.

16. Qazi R, Hameed B. (2002). Bleeding diathesis in acute Myeloid Leukaemia : Thrombocytopenia alone or in Disseminated Intravascular Coagulopathy. Pak $J$ Med Sci, 13, 682-685.

17.Radha R, Minakshi V, Ashok K, Sunita S. (2014). Incidence of acute and chronic forms of leukaemia in Haryana; International Journal of Pharmacy and Pharmaceutical Sciences, 6(2), 42- 45.

18.Rani S, Beohar PC, Mohanty TK, Mathur MD. (1982). Leukaemic pattern in Delhi--a ten-year study of 490 cases. Indian J Cancer, 19(2), 81- 6.

19.Sabina C, Gina Z, Renato B. (2014). Diagnosis and Sub classification of Acute Lymphoblastic Leukaemia . The Mediterranean Journal of Hematology and Infectious Diseases, 6(1), e2014073.

20.Sareen R, Kapil M, Gupta GN. (2018). Incubation and its effect on Leishman stain. J Lab Physicians, 10(03), 357-361.

21.Sharma S, Nangia N, Jain S, Narayan S, Harbhajanka A, Singh S. (2009). Clinico Haematological Profile of Acute Megakaryoblastic Leukaemia: Report of Five Cases. Adv Hematol, 10, 673-6.

22. Singh T. (2018). Atlas and text of Hematology. Avi. Pub, 4 (1), 237-314.

23.Varghese PR, Elayidom NB, Joseph CD, Kumar S. (1984). Epidemiological observations on leukaemia in Kerala (A study of 1016 cases over three years), Ind $J$ Haemato, 2, 15-17.

24. Vasavada PS, Akbar Ali, Mukherjee DP. (1962). Clinical studies in leukaemia - A review of 100 cases in symposium in Leukaemia. Leukaemia Journal of associations Phy India, 9, 357. 